\title{
SIMULTANEOUS SPECTROSCOPY AND POLARIMETRY OF Be STARS
}

D. R. Gies

McDonald Observatory, The University of Texas at Austin

David McDavid

Division of Earth and Physical Sciences, The University of Texas at San Antonio

Evidence is now accumulating that many Be stars display photospheric line profile variations on timescales of days or less that are probably caused by nonradial pulsations (Baade 1984; Penrod 1986). In some circumstances these pulsations can promote mass loss into the circumstellar envelope, and consequently the conditions in the inner part of the envelope may vary on similar timescales. Changes in the envelope could produce variations in the polarization and emission line profiles, and observers have reported rapid variability in both. We describe here an initial attempt to search for simultaneous variations in continuum polarization, $H \propto$ enission, and the He I $\lambda 6678$ photospheric absorption line in order to investigate correlated changes on short timescales.

We made simultaneous observations of the $\mathrm{H} \alpha$ and $\mathrm{He} I \lambda 6678$ line profiles and the UBVRI polarizations of the Be stars $\gamma$ Cas, $\phi$ Per, 48 Per, $\zeta$ Tau, and HD 45995 over a one-week period in January 1986 at the University of Texas McDonald Observatory, using the Coude Reticon spectrometer with the 82-inch telescope and the Breger polarimeter with the 36-inch telescope. Wide-band polarimetry of each of the program stars was obtained on at least four different nights. Observations of polarized standard stars verify that the instrumental precision is adequately estimated by photon statistics and is on the order of $\sigma=0.02 \%$ for stars of the brightness range observed. The only Be star which did not show variations greater than $3 \sigma$ over four nights was 48 Per. Systematic trends were observed only for $\phi$ Per and $\zeta$ Tau, in which the intrinsic $B$ band polarizations declined from $2.34 \%$ to $2.15 \%$ and from $1.55 \%$ to $1.49 \%$ respectively. Two or three spectra (resolution of 0.558 FWHM) of each star were obtained every night. The $H \alpha$ line varied in all the stars but the changes were largest in $\phi$ Per and $\zeta$ Tau. Both showed increased emission (from 5.20 to 5.42 and from 2.34 to 2.42 , respectively, in units of local continuum intensity) and a shift of the emission peak to the red. Both stars are suspected spectroscopic binaries, and in the case of $\phi$ Per, the changes in the Ha profile structure appear to repeat every orbit, accompanied by some cycle-to-cycle variations in equivalent width (Poeckert 1981). However, the polarimetric data for both stars, even when supplemented by measurements from the past 20 years, show no dependence on orbital phase. 
We do not believe that the decrease in polarization observed in these two stars is due primarily to the dilution of the polarized light (originating mainly from scattering of starlight in the inner region of the circumstellar envelope) by increasing continuum emission from the outer envelope (as indicated by increasing emission line intensity). Based on our observations and the models of McLean (1979), any reasonable estimate of the increase in unpolarized flux from the envelope is too small to account for the measured decrease in polarization. Furthermore, there are several well documented cases in which large changes in polarization have been followed by similar changes in $\mathrm{H} \alpha$ emission strength after a time lag of several weeks (Poeckert et a1. 1979; Hayes and Guinan 1984). Since $\phi$ Per and $\zeta$ Tau both have very strong emission, we suspect that their envelopes are optically thick at $\mathrm{H} \alpha$ and that the variations observed correspond to changes in the outer envelope, while the polarization decreases reflect changes in the inner envelope which is hidden at $\mathrm{H} \alpha$. Correlated short-term variations between the polarization and emission lines are probably only observed in low density, optically thin envelopes.

In most of the stars the He I $\lambda 6678$ photospheric absorption line is genera1ly shallow and distorted by envelope lizne components. A single He I $\lambda 6678$ emission line dominates the profile in the spectrum of $\phi$ Per and appears to follow the velocity curve of the secondary star derived by Poeckert (1981) based only on the double-peaked He II $\lambda 4686$ emission line. The He I $\lambda 6678$ line profile in $\zeta$ Tau is marked by a deep, central shell line which showed little variability. The surrounding line wings, however, displayed significant variations in velocity, possibly with a period of 15.5 hours. We suspect that the variations arise in the photospheric line component and correspond to the moving bump pattern seen in other Be stars which are suspected nonradial pulsators.

\section{$\underline{\text { References }}$}

Baade, D. (1984). There are more absorption line profile-variable Be stars with short periods. Astr. Astrophys., 134, 105-17.

Hayes, D. P. and Guinan, E. F. (1984). The morphology of a mass loss episode of the Be star w Ori. Astrophys. J., 279, 721-8.

McLean, I. S. (1979). Interpretation of the intrinsic polarizations of early-type emission-line stars. Mon. Not. R. Astr. Soc., $186,265-85$.

Penrod, G. D. (1986). Episodic mass motions and surface phenomenon. Pub. Astr. Soc. Pac., 98, 35-6.

Poeckert, R. (1981). A spectroscopic study of the binary Be star $\phi$ Persei. Pub. Astr. Soc. Pac., 93, 297-317.

Poeckert, R. Bastien, P. and Landstreet, J, D. (1979). Intrinsic polarization of Be stars. Astr. J., 84, 812-30. 


\section{DISCUSSION FOLLOWING GIES}

Marlborough:

Did you observe any changes in the position angle of polarization accompanying the changes in the amount of polarization?

Gies:

We observed no significant variation in the position angle of intrinsic polarization for any of our program stars.

Henrichs:

Would you suspect any orbital variations in the polarization of your sample stars?

Gies:

We cannot exclude that possibility because our negative result depends on data from the past 20 years for phase coverage, and the orbital variation could be masked by long-term changes.

Torres:

What are the orbital periods of the binary systems?

Gies:

The periods are 127 and 133 days, respectively, for $\phi$ Per and $\zeta$ Tau.

Buscombe:

In optical spectra of $\phi$ Per at many phases, Elaine M. (Hendry) Halbedel measured absorption lines of the secondary early B-type star (Ph.D., thesis, Northwestern U., 1975). Gies:

Poeckert (1981) has reviewed the early measurements by Hendry and Hynek, and concludes that these workers observed shell lines in the vicinity of the primary. Based on the high excitation associated with the HeII $\lambda 4686$ emission line that traces the motion of the secondary, Poeckert suggests that the companion is a hot, low mass star.

Polidan:

$I U E$ observations of $\phi$ Per show very strong CIV emission at 1548 and $1550 \AA$. This emission clearly does not move with the secondary but is constant in velocity as are the HeI and HeII lines. This is a rather surprising result and shows how complex the line formation regions are in this binary.

Gies:

An origin of the CIV lines in the outflow from the primary Be star would be consistent with observations of this line, in other, single Be stars.

Tarafdar:

Are there sufficient hard photons in Be stars to produce enough HeII and HeIII ions to produce $\mathrm{HeI}$ and HeII lines in emission?

Gies:

Both ionization states are observed in emission in $\gamma$ Cas, as well as $\phi$ Per, so the radiative flux is sufficient in some cases. 